Article

\title{
Design of a Metal 3D Printing Patient-Specific Repairing Thin Implant for Zygomaticomaxillary Complex Bone Fracture Based on Buttress Theory Using Finite Element Analysis
}

\author{
Yu-Tzu Wang ${ }^{1}$, Chih-Hao Chen ${ }^{2}$ (D) Po-Fang Wang ${ }^{2}$, Chien-Tzung Chen ${ }^{2}$ and Chun-Li Lin ${ }^{1, *(1)}$ \\ 1 Department of Biomedical Engineering, National Yang-Ming University, Taipei 11221, Taiwan; \\ doraemon781227@gmail.com \\ 2 Craniofacial Research Center, Department of Plastic and Reconstructive Surgery, Chang Gung Memorial \\ Hospital, Linkou 33305, Taiwan; chchen5027@gmail.com (C.-H.C.); pofang1223@gmail.com (P.-F.W.); \\ ctchenap@cgmh.org.tw (C.-T.C.) \\ * Correspondence: cllin2@ym.edu.tw; Tel.: +886-2-2826-7000 (ext. 7039)
}

Received: 21 April 2020; Accepted: 3 July 2020; Published: 9 July 2020

\begin{abstract}
This study developed a zygomaticomaxillary complex (ZMC) patient-specific repairing thin (PSRT) implant based on the buttress theory by integrating topology optimization and finite element (FE) analysis. An intact facial skeletal (IFS) model was constructed to perform topology optimization to obtain a hollow skeleton (HS) model with the structure and volume optimized. The PSRT implant was designed based on the HS contour which represented similar trends as vertical buttress pillars. A biomechanical analysis was performed on a ZMC fracture fixation with the PSRT implant and two traditional mini-plates under uniform axial loads applied on posterior teeth with $250 \mathrm{~N}$. Results indicated that the variation in maximum bone stress and model volume between the IFS and HS models was $15.4 \%$ and $75.1 \%$, respectively. Small stress variations between the IFS model and repairing with a PSRT implant (2.75-26.78\%) were found for compressive stress at frontal process and tensile stress at the zygomatic process. Comparatively, large stress variations (30.67-96.26\%) with different distributions between the IFS model and mini-plate models were found at the corresponding areas. This study concluded that the main structure/contour design of the ZMC repair implant according to the buttress position and orientation can obtain a favorable mechanical behavior.
\end{abstract}

Keywords: bony supporting; patient matched; 3D printing; topology optimization; finite element method

\section{Introduction}

The objectives of repairing zygomaticomaxillary complex (ZMC) fractures involve stabilization and rehabilitation for craniofacial diseases [1-3]. Titanium implants (mini-plates) that were usually used to obtain superior realignment is the clinical standard treatment procedure in surgical repair [4-6], but the traditional mini-plate provides a fixation function only to the resting bone, but the mid-face stability after surgery is not considered, which may lead to plate deformation or screw loosening, making an asymmetrical mid-face appearance. Understanding the stress/strain biomechanical behavior of the ZMC fracture, the repaired and healing facial skeleton is relevant to the design of fixation implants [7]. However, there is still no consensus on the optimization strategy for designing an internal fixation implant for ZMC fractures. The repair stability of ZMC fractures remains controversial, because the fixation of fractures may not require maximum rigidity or strength to achieve reliable bone healing $[7,8]$. However, proponents of single plate implants believe that decreasing the number of 
plates provides another way to decrease morbidity [8]. Therefore, a better conception of the ZMC fracture repair biomechanics is important for improving internal fixation implant design [9].

The design of bone implants for ZMC fractures must reflect the basic biomechanical foundation of bone stability $[10,11]$. The buttresses are composed of a thick cortical bone to protect the face against external assaults and to take shape of horizontal and vertical pillars in the mid-facial bones, providing anchor points for various plates and fixtures, and playing a fundamental role in facial aesthetics through their influence on facial width and projection [7]. Occlusal forces generated during biting or mastication are transmitted by the zygomatic process zygomaticoalveolar crest onto the maxilla up to the zygomatic bone by three vertical buttresses, i.e., the nasomaxillary, zygomaticomaxillary, and the pterygomaxillary buttresses. Other horizontal buttresses stabilize the vertical buttresses mechanically by interconnecting them at different levels [2]. The distribution of facial skeleton buttresses is an important consideration for the design of fracture repairing implants, and accepts anchor points for plates and screws [12].

Integrating computer aided design (CAD) with 3D printing (additive manufacturing) techniques can predesign and fabricate internal fixation implants for repairing ZMC fractures using patient-specific appearance and functional requirements $[13,14]$. Metal 3D printing patient-specific implants increase the interfacial fit adaption between the reconstruction plate and bone, while servicing thin implants for reduction and fixation. However, the large volume of titanium implants may cause a significant stress shielding effect, which leads to bone resorption [14]. Traditionally, clinical requirements for the ZMC fracture involved resting bone restoration and fixation using a mini-plate after the mid-face reconstruction surgery. However, geometric restoration demands, lightweight structure, and primary stability are increased in the current mid-face fracture treatment protocol. A potential repair ZMC implant design can combine with the topology optimization to suggest the best structural profile to achieve clinical requirement [14-17]. The topology optimization is a numerical method usually integrated with the finite element (FE) analysis for use to optimize the design of structural objects [18]. The occlusal forces were transmitted upward through the nasomaxillary buttress and zygomaticomaxillary buttress to the mid-face. The main supporting structures are found on the mid-face of the intact bone with bone thickness expressed after performing the topology optimization. If the bone plate was designed according to the mid-face main supporting structure, it could replace the intact bone to support and transmit the occlusal forces in the mid-face. This useful tool has been widely used in biomechanical design to optimize the structure of orthopedics and craniofacial implants [14,16].

Considering the fundamental buttress biomechanical basis for skeletal stabilization and designing the ZMC patient-specific repairing thin implant repair based on the optimization structure is necessary (denoted as the PSRT implant). The PSRT implant is essential to maximize the buttress function for force transfer and anchor, while maintaining proper strength to restore patient appearance and the necessary degrees of stabilization for bone healing. The objective of this study was to develop a ZMC PSRT implant based on the buttress theory by integrating topology optimization and FE analysis. The biomechanical analysis was also performed to understand the stress difference for the ZMC fracture repair with the PSRT implant and traditional straight mini-plates.

\section{Material and Methods}

\subsection{FE Model Construction and Topology Optimizations}

The computed tomography (CT) scan images were used to construct a left ZMC comminuted fracture solid model (denoted as the ZMC model) by converting bone contours at different cross-sections in the CAD system (Figure 1a). In order to understand the mechanical behavior for the intact facial skeletal, the left ZMC comminuted fracture was deleted and the right intact part was mirrored to generate a complete intact facial skeletal model (denoted as the IFS model) (Figure 1b). 


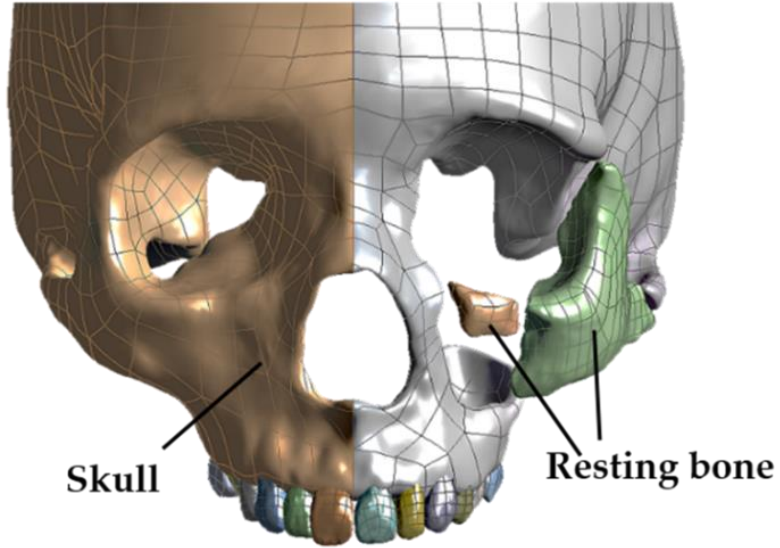

(a)

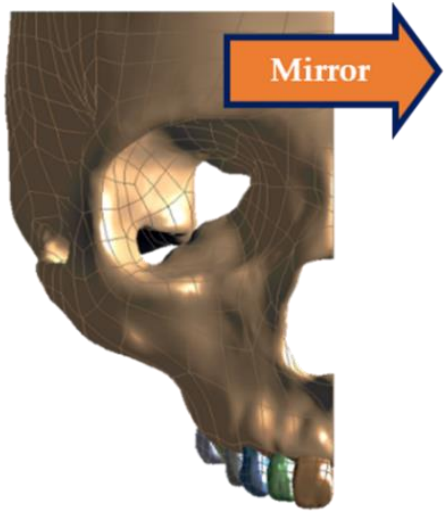

(b)

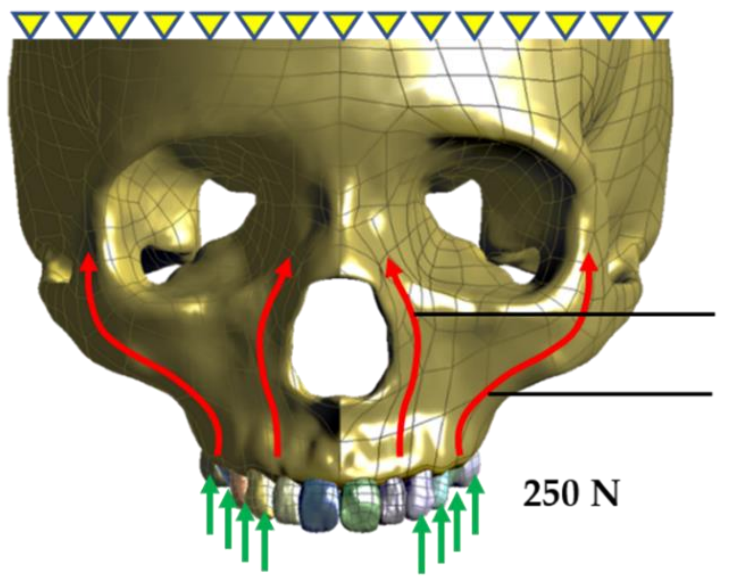

Nasomaxillary buttress

\section{Zygomaticomaxillary buttress}

(c)

Figure 1. Three-dimensional (3D) reconstruction model of the (a) zygomaticomaxillary complex (ZMC) fracture; (b) the right intact part of the ZMC model; (c) a whole intact facial skeletal model (denoted as the IFS model).

The IFS models were exported in the ANSYS Workbench (v19.0, ANSYS Inc., PA, USA) for simulation. The tetrahedral structural elements were used to generate the FE model after mesh convergence tests [19]. Linear elastic material properties that included elastic modulus and Poisson's ratio values were $13,700 \mathrm{MPa} / 0.3$ for cortical and $1370 \mathrm{MPa} / 0.3$ for cancellous bones, respectively [9]. Uniform axial loads were applied on posterior teeth (premolars and molars) with a total of $250 \mathrm{~N}$ as the load conditions. The boundary condition was set as exterior nodes on the frontal and temporal bone segment to fix in all directions (Figure 1c).

The topology optimization modulus in the ANSYS was used to optimize the structure and to reduce the volume of the IFS model. To minimize the IFS model, the volume was defined as the objective function and the following equation was performed:

$$
\left\{\begin{array}{c}
\min V=F\left(\rho_{e}\right) \\
\text { s.t } \sigma_{e} \leq \bar{\sigma}
\end{array}\right.
$$

$\mathrm{V}$ is the volume of design structure, $\rho_{e}$ is the design variable, $\sigma_{e}$ is the von-Mise stress in each element, and $\bar{\sigma}$ is the maximum allowable stress. $\min V=F\left(\rho_{e}\right)$ is the objective function. This function represents the volume $(\mathrm{V})$ that is being minimized for best performance. s.t $\sigma_{e} \leq \bar{\sigma}$ is the characteristic 
that the solution needs to be satisfied. The element whose stress is below the minimum allowable stress can be removed.

The Von Mise stress value of $20 \mathrm{MPa}$ was setup as the minimum allowable stress and each element whose stress was below it was removed to generate a hollow skeleton model (denoted as HS) using the model smoothing procedure (Figure 2).

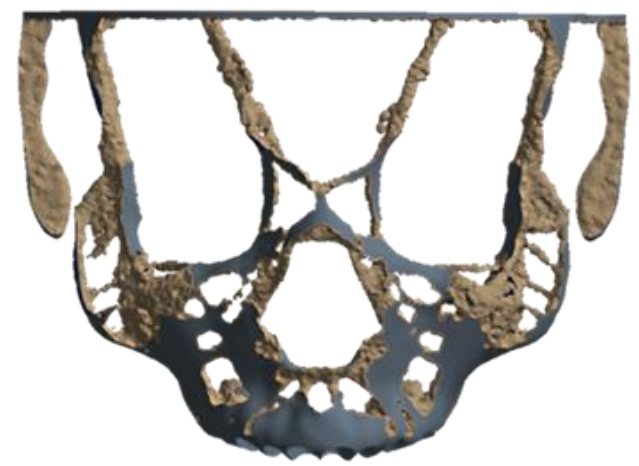

(a)

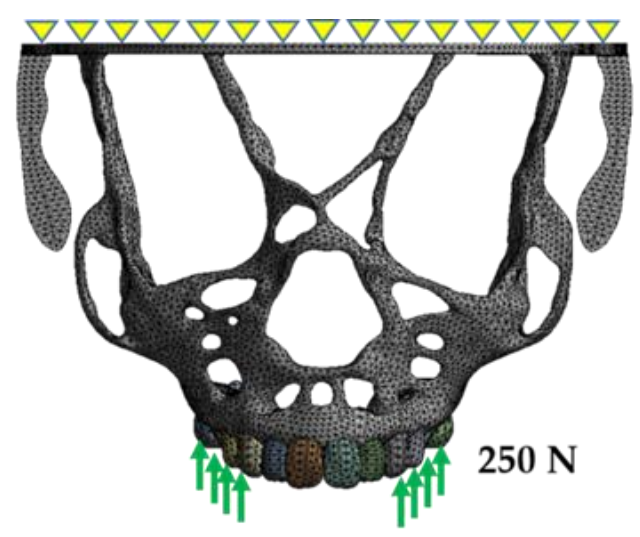

(b)

Figure 2. (a) The hollow skeleton model with jagged appearance obtained from the topology optimization process; (b) model smoothing to refine the jagged boundary profile and generate the optimal structure mesh model with a hollow skeletal (denoted as HS) construction.

\subsection{Buttress Theory Verification by the FE Analysis}

Buttress theory: The supporting facial bony structure can be classified as vertical and horizontal buttresses. The vertical buttresses consist of the paired nasomaxillary (NM) and zygomaticomaxillary (ZM) buttress which can provide the bony support required for mastication and protect the surrounding thin bone [20].

The HS model hollow skeleton structure represents the necessary structure to be retained after IFS model optimization. The other volumes removed are those where the stress value is low and does not need to exist. In order to verify the buttress theory, the same load and boundary conditions from the previous setting were applied on the HS model to compare the variations in mechanical responses between the IFS and HS models (Figure 2a,b).

\subsection{Design of ZMC Patient-Specific Repairing Thin Implant (PSRT Implant)}

The HS model obtained from the topology optimization is the supporting structure for sharing the stress distribution and, moreover, the HS structure profile presented a trend similar to the buttress pillars.

The outer contour of the PSRT implant was designed as a closed loop along the frontal process, infraorbital rim, zygomatic process, alveolar bone, and back to frontal process (Figure 3a). Four small closed inner contours were also generated according to the remaining skeletal structure. Since the infraorbital foramen nerve position and the surgical approach needed to be considered together during the surgical operation, the top three inner closed loops were integrated and the entire thin implant was designed as upper and lower halves using an embedded combination. Six screw holes were designed at the PSRT implant surrounding boundary and can be fixed onto the anatomy's thicker cortical rims (Figure 3b). The main mid-face supporting structure from the HS model was obtained as the design criteria for the PSRT implant design, so that the PRST implant with a lightweight structure could restore the mechanical performance and increase mid-face stability. 


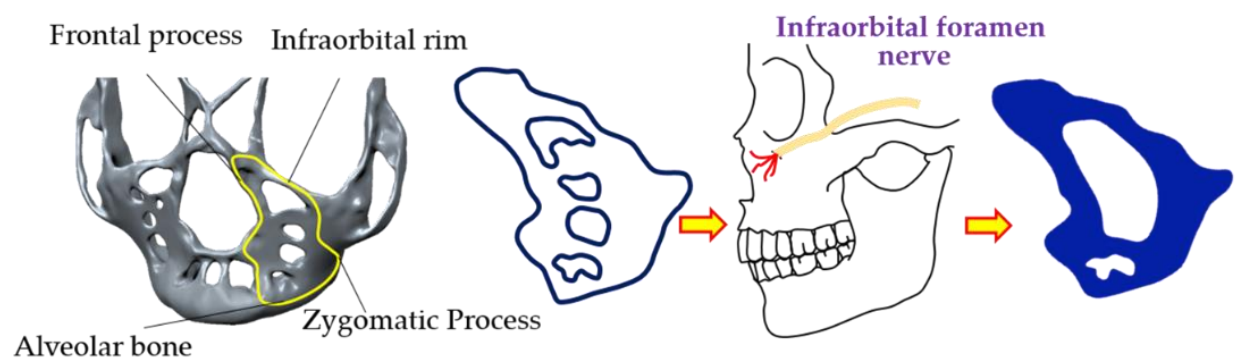

(a)
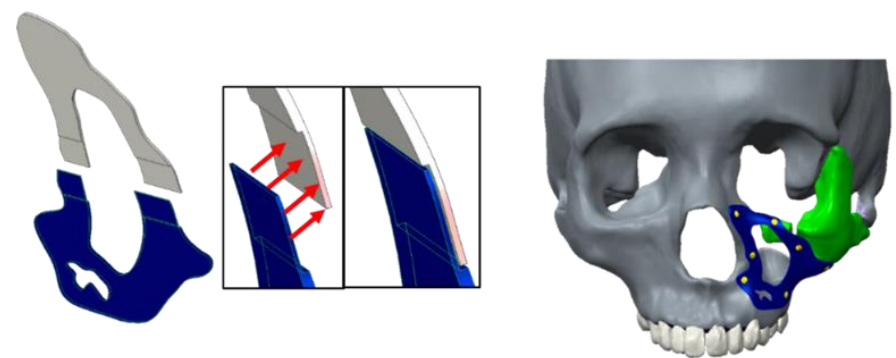

(b)

Figure 3. (a) The patient-specific repairing thin (PSRT) implant contour was generated along the frontal process, infraorbital rim, zygomatic process, alveolar bone, and back to the frontal process. The top three inner closed loops were integrated; (b) a thin implant was designed as the upper and lower halves by an embedded combination. Six screw holes were designed at the PSRT implant surrounding boundary and can be fixed on the anatomy thicker cortical rims.

\subsection{ZMC Fracture Model for the FE Analysis}

Two ZMC comminuted fracture solid models, including fixation with the PSRT implant by six screws surrounding the implant and two traditional mini-plates at the upper and lower parts between the remaining infraorbital rim and zygomatic process bone parts were constructed in the ANSYS. A patient-matched bending thin plate was used in both models to increase the interfacial fit between the plate and orbital wall for repairing an orbital floor fracture (Figure 4) [17]. The stress values of $1 \sim 6$ points were extracted from the HS model and compared with the IFS model, the variation between the two groups was found to be $0.16 \% \sim 11.42 \%$, indicating that the stress distribution of the HS model is similar to the IFS model. After a convergence test with different element sizes [19], FE mesh models were obtained by a smart mesh with quadratic ten-node tetrahedral elements. Nonlinear contact elements with a 0.5 friction coefficient were employed to mimic the interfacial conditions between all screw heads and thin implant/mini-plates in all models (Figure 5) [1,18]. The contact setting between the screw and bone was assumed bonded. Element and node numbers for the patient-specific repair model are 243,615 and 236,605 for the PRST model, and 378,786 and 364,442 for the traditional mini-plate model. Elastic modulus/Poisson's ratio values for titanium alloy for a thin implant/mini-plate were adopted from the literature $[1,19,21]$, i.e., $110 \mathrm{GPa}$ and 0.3 , respectively. The same loading and boundary condition in previous topology optimizations were setup for biomechanical analysis. The maximum and minimum principal stresses for the IFS model and ZMC fracture repair with thin implant/mini-plate models and among variations were recorded. 

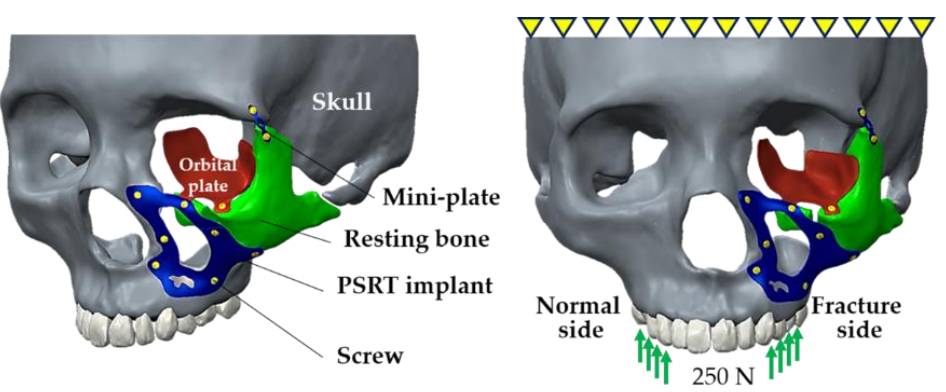

(a)
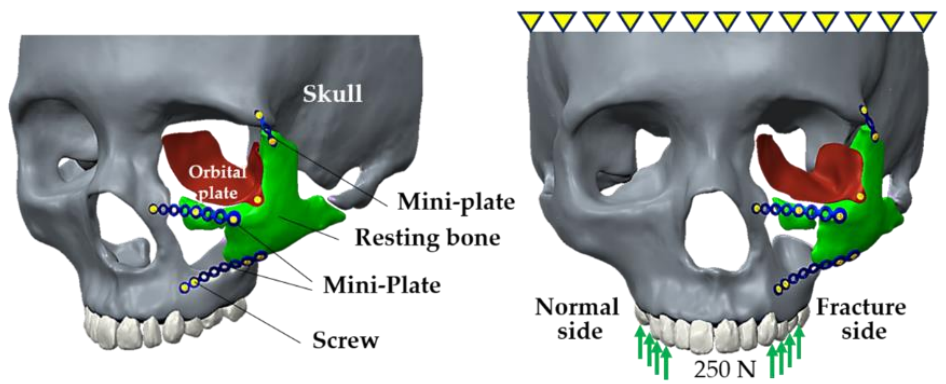

(b)

Figure 4. The facial skeletal ZMC comminuted fracture fixation with (a) the PSRT implant by six screws surrounding the implant and (b) two traditional mini-plates at upper and lower parts between the remaining part of the infraorbital rim and zygomatic process bone were constructed. A patient-matched bending thin plate was used in both models for repairing the orbital floor fracture. Uniform multiple axial loads with a total of $250 \mathrm{~N}$ were applied on premolars and molars as the load condition.

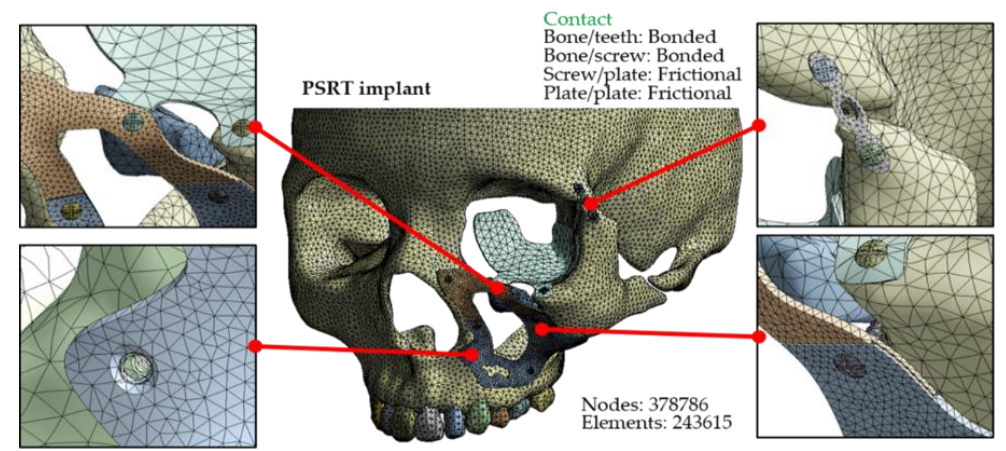

(a)

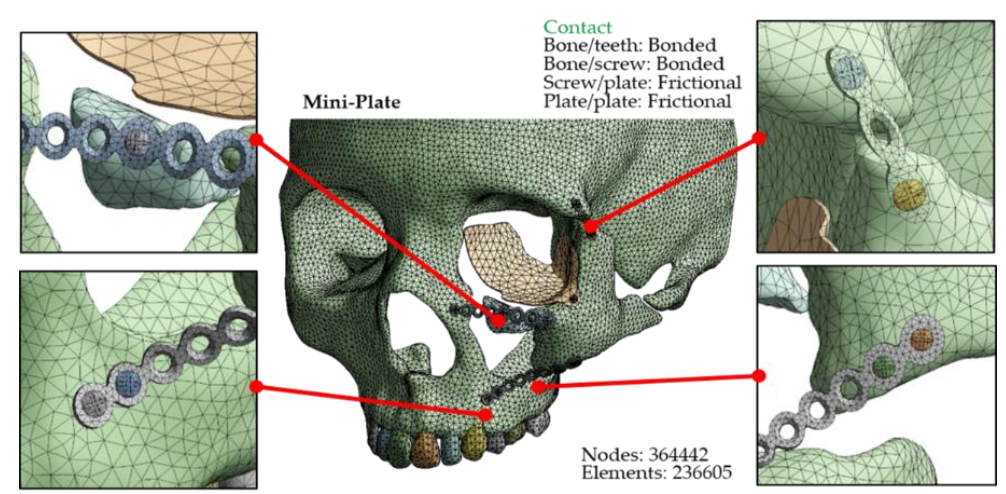

(b)

Figure 5. Finite analysis (FE) mesh models with element/node numbers and interfacial setting conditions for facial skeletal ZMC comminuted fracture fixation with (a) the PSRT implant by six screws surrounding the implant and (b) two traditional mini-plates at upper and lower parts between the remaining part of the infraorbital rim and zygomatic process bone were constructed. 


\section{Results}

The resulting optimal profile of the IFS FE model showed that unnecessary elements were removed under a stress constrain condition and a HS model was generated with jagged appearance to optimize/reduce the structural volume (Figure 2a,b). The HS model structure and high stress regions represented trends similar to the buttress pillars of the nasomaxillary and zygomaticomaxillary buttresses (Figure 6). The variation in maximum bone stress (maximum principal stress) and solid volume between the IFS and HS models was $18.3 \%$ and $75.1 \%$, respectively (Table 1).

\section{$1^{\text {st }}$ Principal stress}

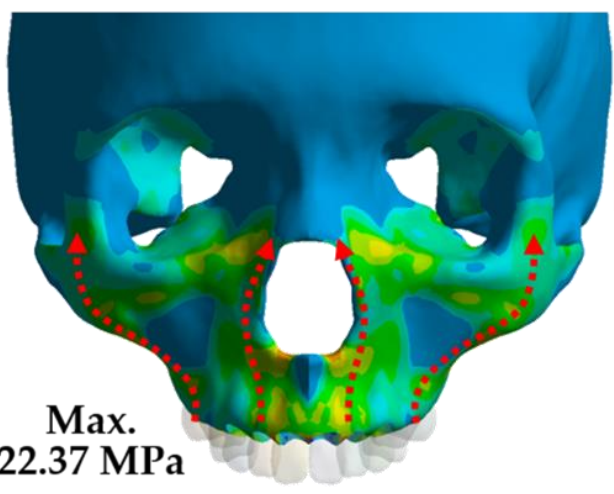

(a) $1^{\text {st }}$ Principal stress

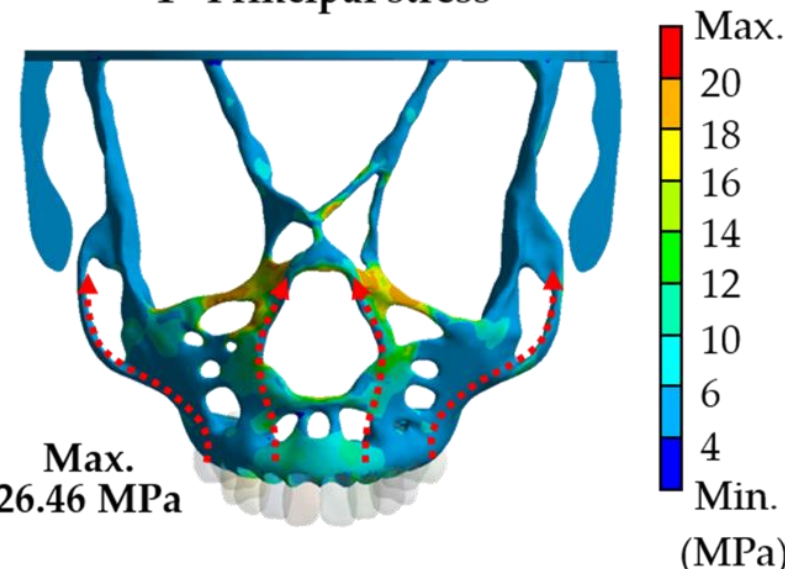

(b)

Figure 6. The stress distribution of the IFS model and HS model, (a) and (b) stress distributions of the IFS and HS models, respectively. High stress regions represented a similar trend as buttress pillars of the nasomaxillary and the zygomaticomaxillary buttresses.

Table 1. The variation of maximum bone stress (maximum principal stress) and solid volume between the IFS and HS models.

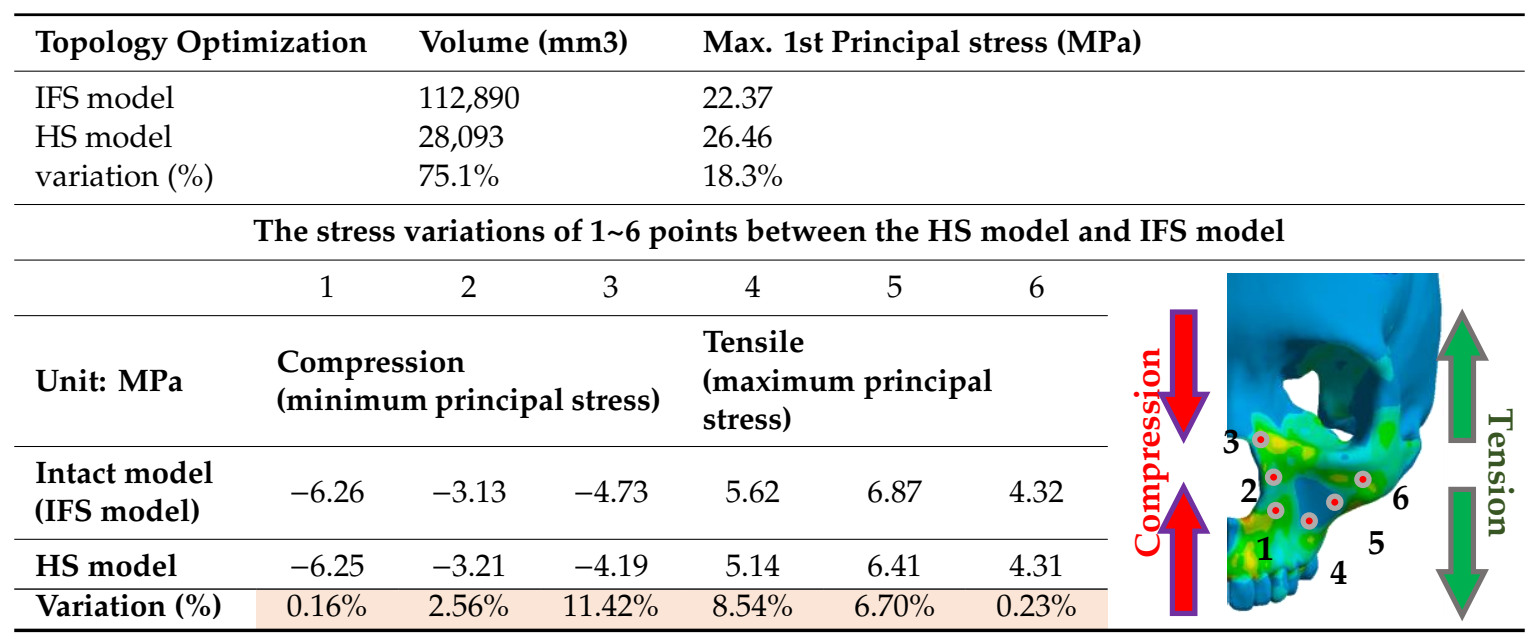

Since the load condition was applied to the vertical force on premolars and molars, it produced a counterclockwise bending moment on the maxillary and zygomatic bones. Points 1, 2, and 3 located at the lower, middle, and upper of the frontal process received compressive stress; the minimum principal stresses (3rd minimum principal stress) on these points were denoted to indicate the magnitude of compressive stresses. The maximum principal stresses (1st maximum principal stress) were denoted to present the tensile stress values of points 4,5 , and 6 located at the lower, middle, upper of the zygomatic process, respectively. Table 2 showed the bone stress values corresponding to points 1 to 6 . Figure 7 shows a comparison of the maximum/minimum bone stress values among the IFS model; the 

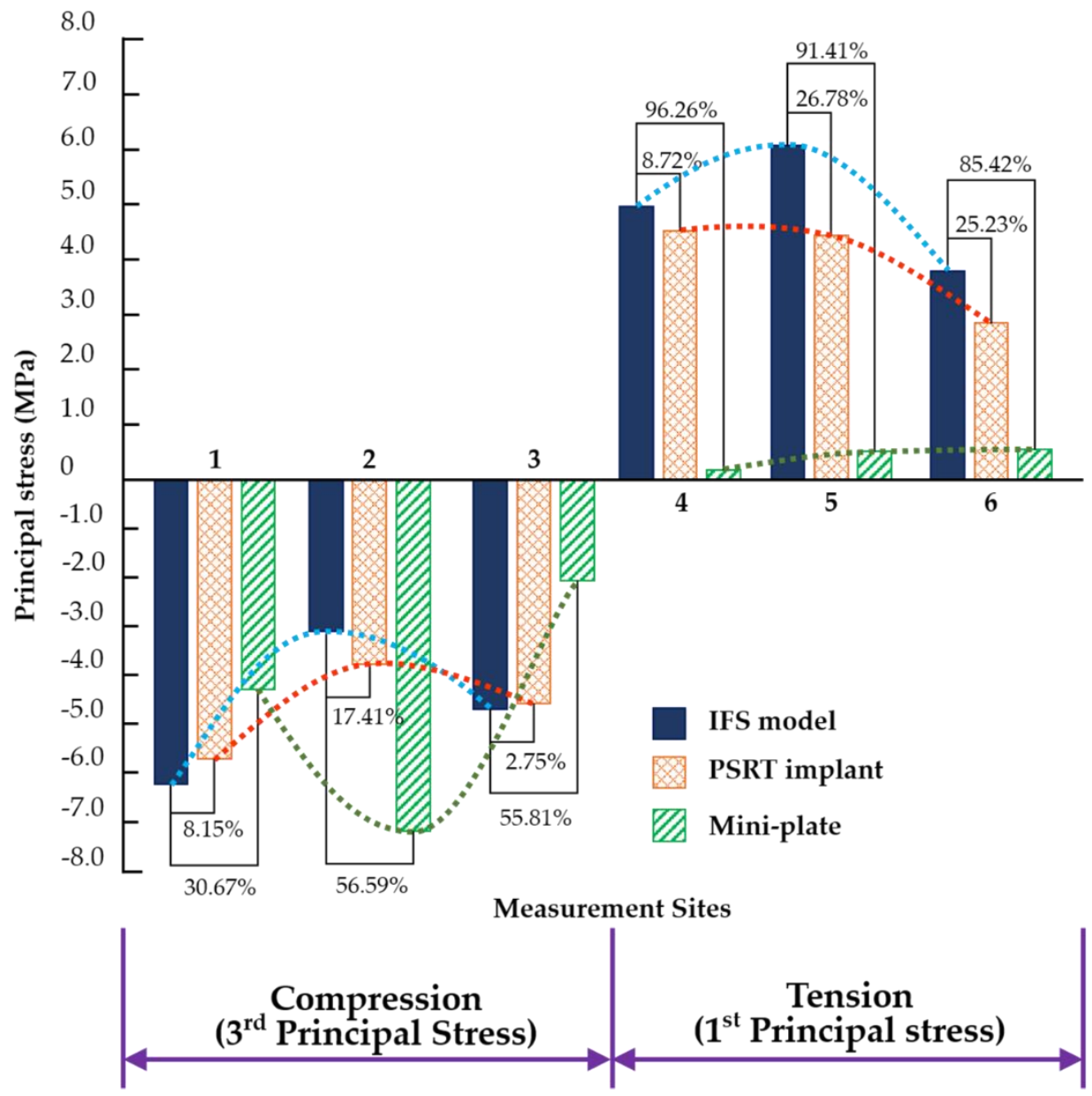

Figure 7. Comparison of the maximum/minimum bone stress value among the IFS model, the ZMC fracture repair with thin implant and traditional mini-plates. A similar stress pattern between the IFS model and repair with the PSRT implant, i.e., convex curves were found while connecting the maximum stress value from points 1 to 3 and points 4 to 6 . However, a different corresponding curve trend was found for repairs with mini-plates from points 1 to 3.
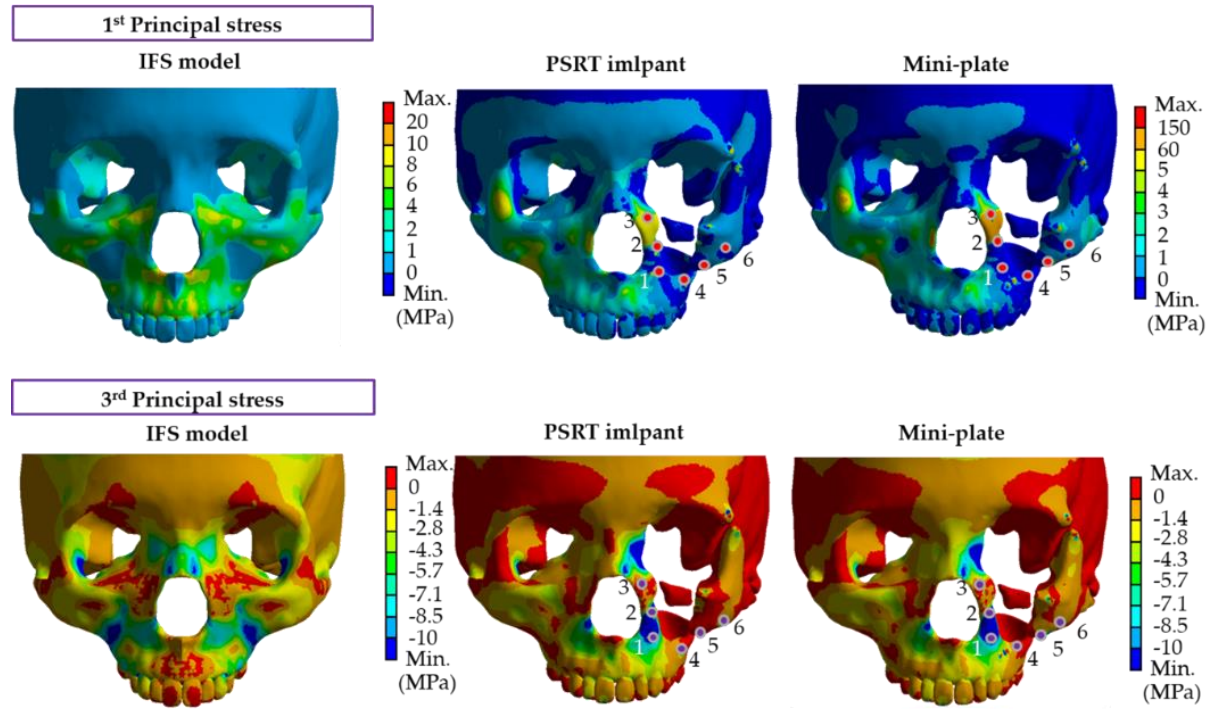

Figure 8. The stress distribution of the intact skeleton bone, the ZMC fracture repairing with the PSRT implant and traditional mini-plates. 


\section{Discussion}

The patient-specific 3D printing fixation implant values applied in plastic surgery or mid-face repair are greatly improved because the implant geometry profile provided an adaptive interfacial fitness, both the positioning and fixation functions for the resting bone. However, patient-specific 3D printing implants must be well designed according to the patient's condition before surgery. However, there is currently no design criterion for applying 3D-printing technology to ZMC fracture repairs. According to the literature and biomechanical principles [2,3,7], the fixation implant needs to be designed as a thin plate, which should not be too rigid to produce a stress-shielding effect. Nor can it be too soft to induce repair instability, and must restore the intact bone mechanical function, i.e., buttress pillars in the mid-facial bones. This is the reason why topology optimization was combined with the FE analysis in the IFS model to verify the generated optimized structure (HS) model as feasible for representing the buttress. The PSRT implant was then designed according to the skeleton of the optimized structure (HS) model.

The topology optimization is a design tool that can arrange a specified number of materials on a given design domain for giving loads, boundary conditions, and constraints to minimize the scalar objective function. This optimization algorithm was combined with the FE analysis in this study to identify optimal structures removing elements whose stress value was less than the constraint value [17]. The objective function was defined to minimize the volume of the intact skeletal structure.

The topology optimization result for the IFS model obtained a HS model with a hollow skeleton structure. The stress pattern that was found through the mid-facial skeleton had similar specific osseous trajectories as the buttresses by occlusal forces. High stress distributions were found at the nasomaxillary and zygomaticomaxillary areas of the HS model and implied that these areas are similar to the position of the former cortical bone with the higher stress registered. The cortical bone with harder stiffness is the main skeleton structure, the adaptive position for screw anchors, and exhibited the highest compressive stress during occlusal. The small stress and large volume variation between the IFS and HS models indicated that many cancellous areas in the facial bone may not need to be transmitted as strength and the structure achieves lightweight neutrality. This point implied that the internal fixation implant contour design can follow the HS model skeleton position, i.e., nearly the buttress pillars in the IFS model.

The maxillary and zygomatic bones received a bending moment under the loading condition in our simulation. Principal stresses were selected more suitable as the stress index of failure for brittle materials rather than von Mises stress. The simulated results clearly showed that the ZMC fracture repair mechanical behavior with the PSRT implant designed according to the HS model skeleton was similar to the IFS model regardless of the stress value and distribution in different positions. However, the use of a traditional mini-plate showed unsatisfactory results. This phenomenon confirms the hypothesis that we proposed the implant main structure or contour design that should be designed according to the buttress position and orientation.

The results obtained in this study were only simulated under the condition of a uniform axial load applied to the posterior teeth, because the chewing condition occurs clinically most often, it was applied to simulate occlusal force. The structural optimization was obtained for explaining the buttress theory under chewing forces. However, this could not represent the actual individual occlusion situations. Well-controlled clinical/animal trials must be confirmed in a further study.

\section{Conclusions}

This study concluded that the topology optimization approach and FE analysis can be integrated to understand the positions of buttress pillars in the mid-face and the main structure/contour design of the ZMC repair implant needed to consider the buttress position and orientation to obtain a favorable mechanical behavior. 
Author Contributions: Conceptualization, C.-T.C., C.-H.C., and P.-F.W.; methodology, Y.-T.W. and C.-L.L.; software, Y.-T.W. and C.-L.L.; investigation, C.-T.C., C.-H.C., and P.-F.W.; resources, C.-T.C., C.-H.C., P.-F.W., and C.-L.L.; writing—original draft preparation, Y.-T.W. and C.-L.L.; writing—review and editing, Y.-T.W., C.-L.L., and C.-T.C.; supervision, C.-L.L. All authors have read and agreed to the published version of the manuscript.

Funding: This research received no external funding.

Acknowledgments: This study is supported in part by MOST 108-2622-B-010 -005 of the Ministry of Science and Technology, Taipei, Taiwan.

Conflicts of Interest: The authors declare no conflict of interest.

\section{References}

1. Balakrishnan, K.; Ebenezer, V.; Dakir, A.; Kumar, S.; Prakash, D. Management of tripod fractures (zygomaticomaxillary complex) 1 point and 2 point fixations: A 5-year review. J. Pharm. Bioallied Sci. 2015, 7, 242-247. [CrossRef] [PubMed]

2. Janovic, A.; Saveljic, I.; Vukicevic, A.; Nikolic, D.; Rakocevic, Z.; Jovicic, G.; Filipovic, N.; Djuric, M. Occlusal load distribution through the cortical and trabecular bone of the human mid-facial skeleton in natural dentition: A three-dimensional finite element study. Ann. Anat. 2015, 170, 16-23. [CrossRef] [PubMed]

3. Pakdel, A.R.; Whyne, C.M.; Fialkov, J.A. Structural biomechanics of the craniomaxillofacial skeleton under maximal masticatory loading: Inferences and critical analysis based on a validated computational model. J. Plast. Reconstr. Aesthet. Surg. 2017, 7, 842-850. [CrossRef] [PubMed]

4. Li, J.P.; Chen, S.L.; Zhang, X.; Chen, X.Y.; Deng, W. Experimental research of accurate reduction of zygomatic-orbitomaxillary complex fractures with individual templates. J. Oral Maxillofac. Surg. 2011, 69, 1718-1725. [CrossRef] [PubMed]

5. Van Hout, W.M.; Van Cann, E.M.; Koole, R.; Rosenberg, A.J. Surgical treatment of unilateral zygomaticomaxillary complex fractures: A 7-year observational study assessing treatment outcome in 153 cases. J. Craniomaxillofac. Surg. 2016, 44, 1859-1865. [CrossRef] [PubMed]

6. Gong, X.; He, Y.; An, J.; Yang, Y.; Huang, X.; Liu, M.; Zhao, Y.; Zhang, Y. Application of a computer-assisted navigation system (CANS) in the delayed treatment of zygomatic fractures: A randomized controlled trial. J. Oral Maxillofac. Surg. 2017, 75, 1450-1463. [CrossRef] [PubMed]

7. Prado, F.B.; Freire, A.R.; Cláudia Rossi, A.; Ledogar, J.A.; Smith, A.L.; Dechow, P.C.; Strait, D.S.; Voigt, T.; Ross, C.F. Review of in vivo bone strain studies and finite element models of the zygomatic complex in humans and nonhuman primates: Implications for clinical research and practice. Anat. Rec. (Hoboken) 2016, 299, 1753-1778. [CrossRef] [PubMed]

8. Wang, Y.T.; Wang, P.F.; Chen, C.T.; Chen, C.H.; Lin, C.L. Biomechanical analysis to verify the buttress theory when using the anatomical thin titanium mesh plate for zygomaticomaxillary complex bone fracture. J. Mech. Med. Biol. 2019, 19, 1-10. [CrossRef]

9. Bluebond-Langner, R.; Rodriguez, E.D. Application of skeletal buttress analogy in composite facial reconstruction. Craniomaxillofac. Trauma Reconstr. 2009, 2, 19-25. [CrossRef] [PubMed]

10. Dai, N.; Zhu, J.; Zhang, M.; Meng, L.Y.; Yu, X.L.; Zhang, Y.H.; Liu, B.Y.; Zhang, S.L. Design of a maxillofacial prosthesis based on topology optimization. J. Mech. Med. Biol. 2018, 18, 1-14. [CrossRef]

11. Wolford, L.M.; Dingwerth, D.J.; Talwar, R.M.; Pitta, M.C. Comparison of 2 temporomandibular joint total joint prosthesis systems. J. Oral Maxillofac. Surg. 2003, 61, 685-690. [CrossRef] [PubMed]

12. Sinno, H.; Tahiri, Y.; Gilardino, M.; Bobyn, D. Engineering alloplastic temporomandibular joint replacements. Mcgill J. Med. 2011, 13, 63-72. [PubMed]

13. Nie, E.M.; Chen, X.Y.; Zhang, C.Y.; Qi, L.L.; Huang, Y.H. Influence of masticatory fatigue on the fracture resistance of the pulpless teeth restored with quartz-fiber post-core and crown. Int. J. Oral Sci. 2012, 4, 218-220. [CrossRef] [PubMed]

14. Pinheiro, M.; Alves, J.L. The feasibility of a custom-made endoprosthesis in mandibular reconstruction: Implant design and finite element analysis. J. Craniomaxillofac. Surg. 2015, 43, 2116-2128. [CrossRef] [PubMed]

15. Lin, C.Y.; Hsiao, C.C.; Chen, P.Q.; Hollister, S.J. Interbody fusion cage design using integrated global layout and local microstructure topology optimization. Spine 2004, 29, 1747-1754. [CrossRef] [PubMed] 
16. Changizi, N.; Kaboodanian, H.; Jalalpour, M. Stress-based topology optimization of frame structures under geometric uncertainty. Comput. Methods Appl. Mech. Eng. 2017, 315, 121-140. [CrossRef]

17. Liu, Y.F.; Fan, Y.Y.; Jiang, X.F.; Baur, D.A. A customized fixation plate with novel structure designed by topological optimization for mandibular angle fracture based on finite element analysis. Biomed. Eng. Online 2017, 16, 131-147. [CrossRef] [PubMed]

18. Seebach, M.; Theurer, F.; Foehr, P.; Deimling, C.; Burgkart, R.; Zaeh, M.F. Advances in Structural and Multidisciplinary Optimization. Design of Bone Plates for Mandibular Reconstruction Using Topology and Shape Optimization, 1st ed.; Springer: Munich, Germany, 2018; pp. 2086-2094.

19. Wang, Y.T.; Chen, C.H.; Wang, P.F.; Lin, C.L. Development of a novel anatomical thin titanium mesh plate with reduction guidance and fixation function for Asian zygomatic-orbitomaxillary complex fracture. J. Craniomaxillofac. Surg. 2018, 46, 547-557. [CrossRef] [PubMed]

20. Stanley, R.B., Jr. Buttress fixation with plates. Oper. Tech. Otolayngol. Head Neck Surg. 1995, 6, 97-103. [CrossRef]

21. Nagasao, T.; Nakajima, T.; Kimura, A.; Kaneko, T.; Jin, H.; Tamaki, T. The dynamic role of buttress reconstruction after maxillectomy. Plast. Reconstr. Surg. 2005, 115, 1328-1340. [CrossRef] [PubMed]

(C) 2020 by the authors. Licensee MDPI, Basel, Switzerland. This article is an open access article distributed under the terms and conditions of the Creative Commons Attribution (CC BY) license (http://creativecommons.org/licenses/by/4.0/). 